\title{
ON PTÁK'S DOUBLE-LIMIT THEOREMS
}

\author{
by N. J. YOUNG \\ (Received 8th August 1969-Revised 14th December 1970)
}

\section{Introduction}

Consider uniform spaces $X$ and $Y$ and a separately uniformly continuous real-valued function $f$ on $X \times Y$. The following question arises in the theory of games: under what conditions can $f$ be extended to a separately continuous function on $\hat{X} \times \hat{Y}$, where $\hat{X}, \hat{P}$ are the completions of $X$ and $Y$ respectively? Firstly observe that such an extension is not always possible. If $X=Y=(0,1]$ with the usual uniform structure and $f(x, y)=x^{y}$ then $f$ is separately uniformly continuous but has no separately continuous extension to $\hat{X} \times \hat{Y}=[0,1]^{2}$ since such an extension would satisfy $f(0,)=$.0 on $Y$ and $f(., 0)=1$ on $X$ and so would necessarily have a discontinuity in one argument at the origin.

A similar problem was solved by V1. Pták (6). If $X$ and $Y$ are completely regular Hausdorff spaces, $C^{\infty}(X)$ and $C^{\infty}(Y)$ are the $B$-spaces of bounded continuous functions on $X, Y$ with supremum norm and $C^{\infty}(X)^{\prime}, C^{\infty}(Y)^{\prime}$ are their duals, Pták obtained a necessary and sufficient condition of a combinatorial character for a bounded real-valued separately continuous function on $X \times Y$ to have a separately $w^{*}$-continuous bilinear extension to $C^{\infty}(X)^{\prime} \times C^{\infty}(Y)^{\prime}$, where we regard $X$ and $Y$ as subsets of $C^{\infty}(X)^{\prime}, C^{\infty}(Y)^{\prime}$ in the canonical way. Since the Stone-Cech compactifications $\beta X, \beta Y$ are the $w^{*}$-closures of $X$ and $Y$ in these duals this immediately gives a criterion for the function to have a separately continuous extension to $\beta X \times \beta Y$. Note that this is a special case of the problem posed above since $\beta X$ is the completion of $X$ under the uniformity it induces on $X$.

Ptak's method is based on his combinatorial lemma on the existence of convex means, from which he also obtains a criterion for the weak compactness of a set in a Banach space, together with various other theorems in which combinatorial assumptions yield topological conclusions. In this paper we use a different approach, based on a theorem of Grothendieck, emphasizing topology rather than combinatorics and obtaining more general results. The major theorem of the paper is the generalized version of Pták's bilinear extension theorem ( $\$ 4)$; we illustrate the use of this result by proving a theorem on the inversion of the order of repeated integrals with respect to finitely additive set-functions.

The author is grateful to the referee for some important observations which led to a recasting of this paper.

$$
\text { E.M.S. -N }
$$




\section{The extension theorem}

Let $\left(z_{i j}\right)$ be a double sequence in a topological space. A point $z$ of the space is said to be a double cluster point (point doublement adherent) of the double sequence if every neighbourhood of $z$ meets infinitely many rows and columns of the sequence, each in an infinite number of points. Theorem 2 of Grothendieck's paper (4) contains the following:

Let $E_{1}$ be a dense subspace of the topological space $E$ and let $F$ be a completely regular space. Let $A$ be a family of continuous functions from $E$ into $F$ with the property that for every sequence $\left(x_{i}\right)$ of members of $E_{1}$ and for every sequence $\left(f_{j}\right)$ of members of $A$ the double sequence $\left(f_{j}\left(x_{i}\right)\right)$ has a double cluster point in $F$. Then every mapping from $E$ into $F$ which is a pointwise limit of members of $A$ is continuous. Consequently if $\{g(x): g \in A\}$ is relatively compact in $F$ for every $x \in E$ then $A$ is relatively compact in the space $C(E, F)$ of continuous mappings from $E$ into $F$ with the topology of pointwise convergence.

The proof is quite elementary. The second statement is not given in quite this form, but it is easily seen to follow from the first part as $A$ is relatively compact in $F^{E}$ by Tychonov's theorem. From this we shall deduce the required extension theorem via the following:

Lemma 1. Let $Y$ be a dense subspace of a topological space $\hat{Y}$, let $E$ be compact and let $F$ be a Hausdorff space. In the following commutative diagram $\lambda$ is the injection of $Y$ into $\hat{Y}, \mu, v$ and $\pi$ are continuous and $v$ is injective.

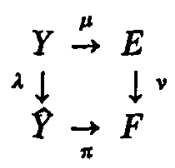

Then $\operatorname{Im} \pi \subseteq \operatorname{Im} v$ and $\mu$ can be extended to a continuous map of $\widehat{Y}$ into $E$.

Proof. Pick any $\pi(y) \in \operatorname{Im} \pi$, so that $y \in \widehat{Y}$. Let $\phi$ be a filter on $Y$ converging to $y$; then by continuity $\pi \circ \lambda(\phi)$ is a filter base on $F$ converging to $\pi(y)$. Now $\mu(\phi)$ is a filter base on $E$, and so by compactness has a cluster point $z \in E$. Thus $v(z)$ is a cluster point of $v \circ \mu(\phi)=\pi \circ \lambda(\phi)$. Hence $\pi(y)=v(z) \in \operatorname{Im} v$. Moreover since $E$ is compact and $F$ Hausdorff $v$ is a homeomorphism of $F$ with $\operatorname{Im} v$; thus $v^{-1} \circ \pi$ is a continuous extension of $\mu$ mapping $\widehat{Y}$ into $F$.

Theorem 1. Let $\hat{X}, \hat{Y}$ be topological spaces and let $Z$ be a compact Hausdorff space. Let $X, Y$ be dense subspaces of $\hat{X}, \hat{Y}$ respectively and let $f: X \times Y \rightarrow Z$ be a separately continuous function such that for every pair of sequences $\left(x_{i}\right)$ in $X,\left(y_{j}\right)$ in $Y$ the double sequence $\left(f\left(x_{i}, y_{j}\right)\right)$ has a double cluster point in $Z$. Then if $f(x,$.$) ,$ $f(., y)$ have continuous extensions to $\hat{Y}, \hat{X}$ for every $x \in X, y \in Y$ respectively it follows that $f$ has separately continuous extension $f^{\prime}: \widehat{X} \times \widehat{P} \rightarrow Z$.

Proof. For any $y \in Y$ let $f_{1}(., y): \widehat{X} \rightarrow Z$ be the continuous extension of $y f(.$,$) . For every x \in \hat{X}$ the function $f_{1}(x,):. Y \rightarrow Z$ is a pointwise limit of 
members of the family $f(X,)=.\{f(x,):. x \in X\}$, and so by the first part of Grothendieck's theorem is continuous; thus $f_{1}: \hat{X} \times Y \rightarrow Z$ is separately continuous and extends $f$. Likewise we may construct a separately continuous function $f_{2}: X \times \hat{Y} \rightarrow Z$ which extends $f$. Define a map $\mu: Y \rightarrow C(X, Z)$ by $\mu(y)=f_{1}(., y)$. In the lemma above take $E$ to be the closure in $C(X, Z)$ of the image $f_{1}(., Y)$ of $\mu$; then $E$ is compact by a direct application of Grothendieck's theorem. Let $F=C(X, Z)$, let $v$ be the restriction map, and let

$$
\pi(y)=f_{2}(., y) \text { for } y \in \hat{Y} \text {. }
$$

Then the hypotheses of the lemma are satisfied and so $\mu$ has a continuous extension $\mu^{\prime}: \hat{Y} \rightarrow B$. Define $f^{\prime}(x, y)$ to be $\mu^{\prime}(x)(y)$ for $x \in \hat{X}, y \in \hat{Y}$. By construction $f^{\prime}$ is separately continuous and extends $f$.

The statement of the theorem is simpler in the case of uniform spaces. Recall that if $X$ is a uniform space with completion $X$ and $Z$ is a complete uniform space then any uniformly continuous function $g: X \rightarrow Z$ has a continuous extension to $\hat{X}$.

Corollary 1. Let $X, Y$ and $Z$ be separated uniform spaces with $Z$ compact. Let $\hat{X}, \hat{Y}$ be the completions of $X, Y$ respectively and let $f: X \times Y \rightarrow Z$ be a separately uniformly continuous function which satisfies the double cluster point condition of the theorem. Then fhas a separately continuous extension to $\hat{X} \times \hat{Y}$.

If $Z$ is sequentially compact the condition on double cluster points can be expressed more simply. Suppose $\left(f\left(x_{i}, y_{j}\right)\right)$ has no double cluster point in $Z$. By use of the diagonal procedure one can replace $\left(x_{i}\right),\left(y_{j}\right)$ by subsequences so that

exist.

$$
\lim _{i} f\left(x_{i}, y_{j}\right)=a_{j} \text { and } \lim _{j} f\left(x_{i}, y_{j}\right)=b_{i}
$$

Then taking further subsequences we may suppose that $a=\lim a_{j}$ and $b=\lim b_{i}$ exist; however $a$ and $b$ must be unequal or their common value would be a double cluster point. Thus the condition of the theorem can be expressed as follows (the converse implication being immediate): if $\lim \lim f\left(x_{i}, y_{j}\right)$ and $\lim _{j} \lim _{i} f\left(x_{i}, y_{j}\right)$ both exist for any pair $\left(x_{i}\right),\left(y_{j}\right)$ of sequences in $X, Y$ then these $j$
repeated limits are equal. If $f$ has this property we shall say that $f$ satisfies the repeated limit condition.

Corollary 2. Let $X$ and $Y$ be completely regular spaces and let $Z$ be a compact and sequentially compact Hausdorff space. Then a separately continuous function $f: X \times Y \rightarrow Z$ has a separately continuous extension $f^{\prime}: \beta X \times \beta Y \rightarrow Z$ if and only if $f$ satisfies the repeated limit condition.

This result (with $Z \subseteq R$ ) was given by Pták (6, Theorem 5.2). Similarly one can show that if $Z$ is realcompact ((2)) then the double cluster point con- 
dition suffices for $f$ (as above) to have a separately continuous extension to $v X \times v Y$ where $v X, v Y$ are the Hewitt realcompactifications of $X, Y$.

An interesting feature of the necessary and sufficient condition in Corollary 2 is that it makes no mention of the topology of $X$ or $Y$; thus $f$ has separately continuous extension to $\beta X \times \beta Y$ if and only if it has separately continuous extension to $\alpha X \times \alpha Y$ where $\alpha X, \alpha Y$ are the Stone-Čech compactifications of $X$ and $Y$ in their discrete topologies. One can further deduce, for example, that the family of all bounded real-valued functions on $X \times Y$ which satisfy the repeated limit condition is complete with respect to the supremum norm and is closed under algebraic operations-facts which are not trivial to verify directly. However this family is not closed under the taking of monotone limits whenever both $X$ and $Y$ are infinite, as one may then construct a countable set in $X \times Y$ whose characteristic function does not satisfy the repeated limit condition.

\section{A criterion for weak compactness}

Another important result obtained by combinatorial methods in Pták's paper (7) is a criterion for weak compactness in Banach spaces: the corresponding result for complete locally convex spaces is given by Grothendieck (4, Theorem 7). Here we present a strengthening of this criterion.

If $(E, F)$ is a dual pair of real or complex vector spaces and $A \subseteq E, B \subseteq F$ then we say that $A \times B$ satisfies the repeated limit condition if for all sequences $\left(x_{i}\right),\left(y_{j}\right)$ taken from $A, B$ respectively we have

$$
\lim _{i} \lim _{j}\left\langle x_{i}, y_{j}\right\rangle=\lim _{j} \lim _{i}\left\langle x_{i}, y_{j}\right\rangle
$$

whenever both repeated limits exist. It is clear that if $A$ and $B$ are relatively compact in $\sigma(E, F)$ and $\sigma(F, E)$ then $A \times B$ satisfies the repeated limit condition. We denote the bipolar of a set $A \subseteq E$ by $A^{00}$; thus $A^{00}$ is the $\sigma(E, F)$-closed absolutely convex hull of $A$ in $E$.

Theorem 2. If $(E, F)$ is a dual pair of real or complex vector spaces and $A, B$ are subsets of $E, F$ such that $\{\langle x, y\rangle: x \in A, y \in B\}$ is bounded and $A \times B$ satisfies the repeated limit condition then the same holds for $A^{00} \times B$.

In the case where $B$ is absolutely convex and weakly compact this conclusion may be found in (5, p. 332), where a combinatorial proof (again due to Pták) is given, and in the proof of Theorem 7 of (4). The present proof is based on the latter.

Proof. Let $F_{1}$ denote the linear subspace of $F$ generated by $B$. Then $A$ is a $\sigma\left(E, F_{1}\right)$-bounded set and hence $(8$, p. 50$)$ is a precompact (though possibly not separated) space in the uniform structure induced by $\sigma\left(E, F_{1}\right)$. Let $\hat{A}$ denote the completion of the space obtained by identifying non-separated 
points of $A$; then every member of $F_{1}$ (being uniformly continuous on $A$ ) has a continuous extension to $\hat{A}$. Denote this extension by $t y$ for every $y \in F_{1}$, so that $t$ is a linear transformation from $F_{1}$ into the space $C(\hat{A})$ of continuous real-valued functions on the compact Hausdorff space $A$. By Grothendieck's theorem (\$2) $t(B)$ is relatively compact in $C(\hat{A})$ in the topology of pointwise convergence, and by hypothesis $t(B)$ is bounded. Hence by a further theorem of Grothendieck (4, Theorem 5$) t(B)$ is relatively weakly compact. Let $K$ denote the unit ball of the Banach dual $C^{\prime}(\hat{A})$ of $C(\hat{A})$ (taken with respect to the supremum norm). Then $K$ is $w^{*}$-compact and hence $t(B) \times K$ satisfies the repeated limit condition. Now let $F_{1}^{*}$ denote the algebraic dual of $F_{1}$ and let

$$
t^{\prime}: C^{\prime}(\widehat{A}) \rightarrow F_{1}^{*}
$$

be the adjoint of $t$. Then it follows that $B \times t^{\prime}(K)$ satisfies the repeated limit condition in $\left(F_{1}, F_{1}^{*}\right)$. Since $t^{\prime}$ is continuous with respect to $\sigma\left(F_{1}^{*}, F_{1}\right)$ and the $w^{*}$-topology on $C^{\prime}(\hat{A})(8$, p. 38$), t^{\prime}(K)$ is a $\sigma\left(F_{1}^{*}, F_{1}\right)$-compact absolutely convex set. Moreover $t^{\prime}(K)$ contains the image $i(A)$ of $A$ under the canonical mapping $i$ of $E$ into $F_{1}^{*}$. This mapping is continuous with respect to the topologies $\sigma\left(E, F_{1}\right)$ and $\sigma\left(F_{1}^{*}, F_{1}\right)$ and hence $i^{-1} t^{\prime}(K)$ is a $\sigma\left(E, F_{1}\right)$-closed absolutely convex set containing $A$, and clearly $i^{-1} t^{\prime}(K) \times B$ satisfies the repeated limit condition. The topology $\sigma(E, F)$ is finer than $\sigma\left(E, F_{1}\right)$ so $A^{00} \subseteq i^{-1} t^{\prime}(K)$ and hence $A^{00} \times B$ satisfies the condition.

Theorem 3. Let $(E, F)$ be a dual pair of real or complex vector spaces and let $\mathscr{B}$ be a family of weakly bounded subsets of $F$ whose bipolars generate $F$ algebraically. If $E$ is complete in the topology of uniform convergence on members of $\mathscr{B}$ and $A \subseteq E$ is a $\mathscr{B}$-bounded set such that $A \times B$ satisfies the repeated limit condition for every $B \in \mathscr{B}$ then $A$ is weakly relatively compact.

We observe that Grothendieck (4, Theorem 7 ) makes the additional assumption that the bipolars of members of $\mathscr{B}$ are weakly compact (so that the $\mathscr{B}$ topology is a topology of the dual pair $(E, F)$ ). In that case the condition of the theorem is clearly also necessary.

Proof. Regard $E$ as the space of weakly continuous linear functionals on $F$. Since $A$ is $\mathscr{B}$-bounded it is also weakly bounded and hence by Tychonov's theorem is relatively compact in $\boldsymbol{R}^{F}$ or $\boldsymbol{C}^{F}$ (where $\boldsymbol{R}$ and $\boldsymbol{C}$ denote the real and complex fields). Consequently to prove $A$ relatively weakly compact we need only show that any (real or complex-valued) function $f$ on $F$ which is a pointwise limit of members of $A$ belongs to $E$. Such a function is clearly linear, and for any $B$ in $\mathscr{B}$, Theorem 2 and the hypothesis above imply that $A \times B^{00}$ satisfies the repeated limit condition. Hence by the theorem of Grothendieck (\$2) $f$ is weakly continuous on $B^{00}$. However, by a further theorem of Grothendieck (3) the fact that $E$ is complete in the $\mathscr{B}$-topology implies that every linear functional on $F$ which is weakly continuous on every $B^{00}$ (with $B \in \mathscr{B}$ ) belongs to $E$. The conclusion follows. 
We observe that the theorems of Krein and Eberlein are simple consequences of this criterion together with Theorem 2.

\section{The bilinear extension theorem}

Pták's theorem mentioned in the Introduction states that if $X$ and $Y$ are completely regular spaces and $f$ is a bounded real-valued separately continuous function on $X \times Y$ then $f$ has a separately $w^{*}$-continuous bilinear extension to $C^{\infty}(X)^{\prime} \times C^{\infty}(Y)^{\prime}$ if and only if $f$ satisfies the repeated limit condition. In similar vein we have the following result: let $X$ and $Y$ be locally compact Hausdorff spaces and let $C(X), C(Y)$ be the spaces of all continuous functions on $X, Y$ with the topology of compact convergence, having duals $C(X)^{\prime}$ and $C(Y)^{\prime}$. Then a separately continuous real-valued function on $X \times Y$ which is bounded on compact sets can be extended to a separately weakly continuous bilinear functional on $C(X)^{\prime} \times C(Y)^{\prime}$. In fact both statements are special cases of the following theorem.

Theorem 4. Let $X$ and $Y$ be sets and let $\mathscr{A}, \mathscr{B}$ be families of subsets of $X, Y$ which cover $X, Y$ respectively. Let $E, F$ be vector spaces of real-valued functions on $X, Y$ which are bounded on members of $\mathscr{A}, \mathscr{B}$ and which are complete in the topologies of uniform convergence on the members of $\mathscr{A}, \mathscr{B}$. Let the duals of $E, F$ in these topologies be $E^{\prime}, F^{\prime}$. Let $f: X \times Y \rightarrow R$ satisfy $f(x,.) \in F, f(., y) \in E$ for every $x \in X$ and $y \in Y$, and suppose that the restriction of $f$ to $A \times B$ is bounded and satisfies the repeated limit condition for every $A \in \mathscr{A}$ and $B \in \mathscr{B}$. Then $f$ has a separately weakly continuous bilinear extension to $E^{\prime} \times F^{\prime}$.

Proof. Embed $Y$ in $F^{\prime}$ as a set of evaluation functionals. Then we may regard $\mathscr{B}$ as a family of weakly bounded subsets of $F^{\prime}$, and it is simple to check that the bipolars of members of $\mathscr{B}$ span $F^{\prime}$, and the given topology of $F$ is that of $\mathscr{B}$-convergence. For each $A \in \mathscr{A}$ let $A_{1} \subseteq F$ be the set $\{f(x,):. x \in A\}$. The hypothesis on $f$ implies that $A_{1}$ is $\mathscr{B}$-bounded and that $A_{1} \times B$ satisfies the repeated limit condition for each $B \in \mathscr{B}$. Hence by Theorem $3 A_{1}$ is weakly compact. We now require

Lemma 2. Let $Y$ be a set and $F$ a real vector space whose elements are realvalued functions on $Y$ with pointwise operations and whose topology is finer than that of pointwise convergence. Then any continuous linear functional on $F$ can be approximated uniformly on weakly compact subsets of $F$ by linear combinations of evaluation functionals.

For if $F^{\prime}$ denotes the dual of $F$ in the given topology a Hahn-Banach argument shows that the subspace of linear combinations of evaluation functionals is $\sigma\left(F^{\prime}, F\right)$-dense in $F^{\prime}$, and so is dense in any topology of the dual pair $\left(F^{\prime}, F\right)$. The lemma now follows from the Mackey-Arens theorem, which states that the topology of uniform convergence on weakly compact sets of $F$ is such a topology. 
Continuing the proof of the theorem we set $f_{1}(x, v)=\langle f(x,), v$.$\rangle for any$ $x \in X, v \in F^{\prime}$. Clearly $f_{1}(x,$.$) is a weakly continuous linear functional on F^{\prime}$; and for any $A \in \mathscr{A}$ the lemma shows that $f_{1}(., v)$ can be approximated uniformly on $A$ by finite sums $\Sigma \lambda f(., y)$. Thus by the assumption of completeness of $E$ $f_{1}(., v) \in E$.

We may therefore define $f^{\prime}$ on $E^{\prime} \times F^{\prime}$ by $f^{\prime}(\mu, v)=\left\langle f_{1}(., v), \mu\right\rangle$ for $\mu \in E^{\prime}$, $v \in F^{\prime}$. Then $f^{\prime}(., v)$ is linear and weakly continuous on $E^{\prime}$ for each $v \in F^{\prime}$ and $f^{\prime}(\mu,$.$) is linear on F^{\prime}$ for each $\mu \in E^{\prime}$. To show that $f^{\prime}(\mu,$.$) is also weakly$ continuous on $F^{\prime}$ it suffices (on account of the completeness of $F$ ) to show it to be weakly continuous on $B^{00}$ for every $B \in \mathscr{B}$, by the theorem of Grothendieck quoted above.

We first observe that for any $B \in \mathscr{B}$ the set $\left\{f_{1}(., v): v \in B^{00}\right\}$ is contained in $\{f(., y): y \in B\}^{00}$ in $E$; for the argument above shows that if $v \in B^{00}$ then $f_{1}(., v)$ can be approximated uniformly on any $A \in \mathscr{A}$ by absolutely convex combinations $\Sigma \lambda f(., y)$ with $y \in B$. Since $\{f(., y): y \in B\}$ is weakly relatively compact-again by the repeated limit criterion-so also is $\left\{f_{1}(., v): v \in B^{00}\right\}$. Thus any $\mu \in E^{\prime}$ can be approximated uniformly on this set by linear combinations of points of $X$, or equivalently $f^{\prime}(\mu,$.$) can be approximated uniformly$ on $B^{00} \subset F^{\prime}$ by linear combinations $\Sigma \lambda f^{\prime}(x,$.$) . By the definition of f_{1}$ we have $f_{1}(x,)=.f^{\prime}(x,$.$) weakly continuous on F^{\prime}$, so $f^{\prime}(\mu,$.$) is weakly continuous$ on every $B^{00}$.

We remark that the extension $f^{\prime}$ is unique since $X$ and $Y$ generate weakly dense subspaces of $E^{\prime}$ and $F^{\prime}$. Moreover the condition that $f$ satisfy the repeated limit condition on $A \times B$ for every $A \in \mathscr{A}, B \in \mathscr{B}$ is also necessary for the existence of the extension $f^{\prime}$ since $A^{00}$ and $B^{00}$ are weakly compact in $E^{\prime}$ and $F^{\prime}$ and $f$ is a restriction of $f^{\prime}$.

As a consequence of this theorem we obtain

Theorem 5. If $\mu, v$ are bounded additive set-functions on the measurable spaces $(X, \Sigma),(Y, T)$ and $f$ is a bounded separately measurable real-valued function on $X \times Y$ which satisfies the repeated limit condition then $\int_{Y} f(., y) v(d y)$ and $\int_{X} f(x,.) \mu(d x)$ are measurable functions on $X, Y$ respectively and
\[ \int_{X} \int_{Y} f(x, y) v(d y) \mu(d x)=\int_{Y} \int_{X} f(x, y) \mu(d x) v(d y) . \]

The assumption that $f$ be separately measurable means that $f(x,$.$) be$ measurable (T) for every $x \in X$ and $f(., y)$ be measurable $(\Sigma)$ for every $y \in Y$.

Proof. In Theorem 4 take $\mathscr{A}=\{X\}, \mathscr{B}=\{Y\}$ and let $E, F$ be the spaces of bounded measurable functions with respect to $\Sigma, \mathrm{T}$ on $X, Y$. The hypotheses of the theorem are then satisfied and so $f$ has separately weakly continuous bilinear extension $f^{\prime}$ to $E^{\prime} \times F^{\prime}$. $E^{\prime}, F^{\prime}$ are the spaces of bounded additive set-functions 
on $\Sigma, T$ (see $(1$, Theorem IV, 5,1$)$ ), and reference to the proof of Theorem 4 shows that for $\mu \in E^{\prime}, v \in F^{\prime}$ we have

and

$$
f^{\prime}(x, v)=\int_{Y} f(x, y) v(d y)
$$

$$
f^{\prime}(\mu, v)=\int_{X} \int_{Y} f(x, y) v(d y) \mu(d x) .
$$

By symmetry the opposite order of integration must also yield a separately weakly continuous bilinear extension of $f$, and by uniqueness the two must be equal.

This result appears to be new even in the case where $\mu$ and $v$ are measures.

\section{REFERENCES}

(1) N. DUNFord and J. T. SCHWARTz, Linear Operators, Part 1 (Interscience, 1958).

(2) L. Gillman and M. JeRison, Rings of Continuous Functions (Van Nostrand, 1960).

(3) A. Grothendieck, Sur la complétion du dual d'un espace localement convexe, C.R. Acad. Sci. Paris 230 (1950), 605-606.

(4) A. Grothendieck, Critères de compacité dans les espaces fonctionnels généraux, Amer. J. Math. 74 (1952), 168-186.

(5) G. KöTHE, Topologische Lineare Räume I (Springer Verlag, 1960).

(6) VL. PTÁk, An extension theorem for separately continuous functions and its application to functional analysis, Czechoslovak Math. J. 14 (89) (1964), 562-581.

(7) VL. PTÁk, A combinatorial lemma on the existence of convex means and its application to weak compactness, Proc. Sympos. Pure Math. Vol. VII (American Math. Soc., Providence, R.I., 1963), 437-450.

(8) A. P. Robertson and W. J. Robertson, Topological Vector Spaces (Cambridge University Press, 1964).

THE UNIVERSITY

Glasgow, W.2 